\section{Les CAR-T cells, des cellules tueuses spécifiques d'antigènes tumoraux}

\section{De nouvelles générations pour le traitement des tumeurs solides}

Véronique Catros

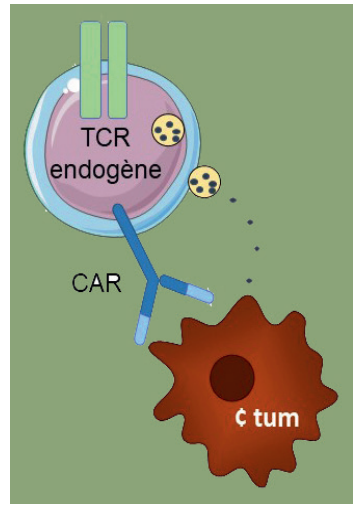

Univ Rennes, Inserm, CHU Rennes, Institut NUMECAN (Nutrition, Metabolisms and (ancer), CRB Santé Rennes, CHU Pontchaillou, 2, rue Henri Le Guilloux, F-35000 Rennes, France.

veronique.catros@univ-rennesl.fr d'autres CAR dirigés, eux, contre des tumeurs solides. De nouvelles générations de CAR visent à mieux contrôler leur prolifération et à améliorer leurs fonctions in vivo grâce à la mise en place de mécanismes d'inactivation inductibles. Le développement des multi-CAR, des CAR spécifiques de plusieurs cibles, et leur combinaison aux inhibiteurs de points de contrôle immunitaires ouvrent une nouvelle ère pour l'immunothérapie des tumeurs. <

\section{Le lymphocyte $T$, une arme efficace}

Les progrès réalisés au cours de ces deux dernières décennies ont établi le rôle essentiel du système immunitaire dans la surveillance et l'élimination des cellules cancéreuses de l'organisme (Figure 1). Après différentes étapes dont l'évaluation clinique de l'activité anti-tumorale de cytokines comme l'interleukine 2 (IL2) ou de lymphocytes activés dès les années 1980, c'est

Vignette (Photo @ Véronique Catros). surtout depuis 2010 que les immunothérapies ont montré leur potentiel clinique. Les anticorps spécifiques de points de contrôle immunitaires (ICP, immune checkpoints), qui ciblent des molécules comme CTLA-4 (cytotoxic T lymphocyte-associated antigen-4) et PD-1 (programmed cell death-1 1$)^{1}$ ont ainsi bénéficié d'autorisations de mise sur le marché (AMM) pour plusieurs types de tumeurs hématologiques ou solides [1]. Certaines de ces tumeurs, comme les mélanomes, répondent à ces thérapeutiques. D'autres, comme les tumeurs pancréatiques, y sont en revanche réfractaires.

Ces anticorps sont en évaluation clinique dans le cadre de nouvelles combinaisons thérapeutiques et de nouveaux anticorps ciblant d'autres molécules impliquées dans le contrôle de l'activation des lymphocytes T, comme TIM-3 ( $T$-cell immunoglobulin and mucincontaining protein-3), TIGIT (T cell immunoreceptor with Ig and ITIM domains), Lag-3 (lymphocyte activation gene 3 ) et $\mathrm{B} 7-\mathrm{H}$, un homoloque de B7.1/B7.2 ou CD80/86 [2, 3] $(\rightarrow)$ sont en cours de développement $[4,5]$. Ces ICP, qui révolutionnent déjà la prise en charge de certains patients atteints de cancer, ne sont en réalité que les révélateurs du potentiel de l'immunité contre le «soi »[6]. En effet, lorsque les mécanismes

$(\rightarrow)$ Voir les Synthèses de V. Catros et al., $m / s n^{\circ} 5$, mai 2014, page 537 et de C. Granier et al., $\mathrm{m} / \mathrm{s}$ $n^{\circ} 3$, mars 2018, page 231

${ }^{1}$ Dont la découverte du rôle immuno-suppresseur de la réponse anti-tumorale par James Allison et Tasuku Honjo vient de faire l'objet du prix Nobel de Physiologie ou Médecine 2018. 


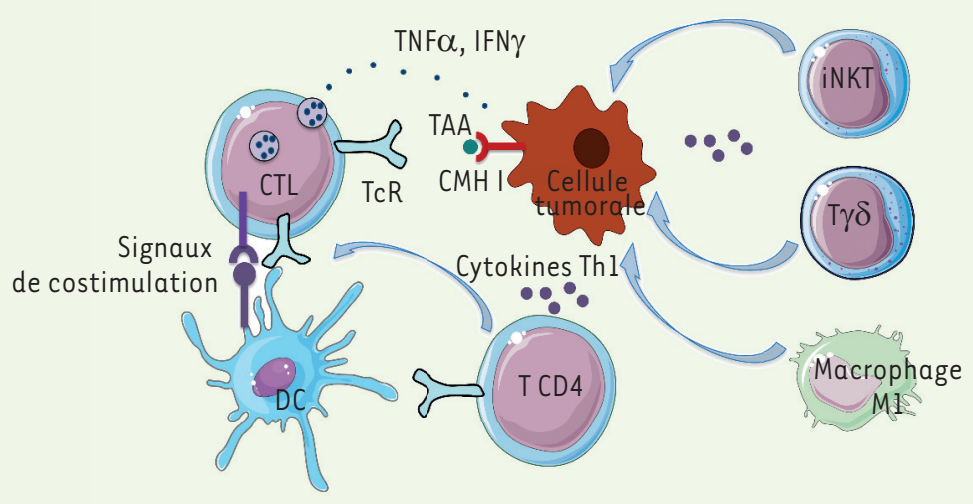

Figure 1. Les cellules effectrices du système immunitaire reconnaissent et tuent les cellules tumorales. Lors d'une réponse immunitaire idéale, les cellules dendritiques (DC) présentent les antigènes aux lymphocytes T CD4 et CD8 dans un contexte de molécules de co-stimulation et d'environnement cytokinique favorable à la différenciation des cellules cytotoxiques (CTL). Les cellules de l'immunité innée (macrophages de type Ml), les cellules iNKT (invariant natural killer $T$ ) et les lymphocytes $T \gamma \delta$ participent à cet environnement. Les CTL reconnaissent des peptides dérivés d'antigènes associés aux tumeurs (TAA) présentés par les molécules de classe I du complexe majeur d'histocompatibilité (CMH) et lysent les cellules tumorales grâce à leurs molécules effectrices (perforine, granzymes) et à la sécrétion de cytokines comme le TNF.

à l'origine de leur blocage fonctionnel sont inhibés (en l'occurrence par les anti-ICP), les lymphocytes $T$ montrent toute leur capacité à reconnaître et à lyser les cellules tumorales [7]

$(\rightarrow)$. Ces cellules effectrices ont en effet pour fonction de maintenir l'intégrité du « soi » en éliminant les cellules anormales. Elles peuvent

$(\rightarrow)$ Voir la Synthèse de V. Catros et al., $\mathrm{m} / \mathrm{s}$ $n^{\circ} 1$, janvier 2003, page 43 néanmoins également être à l'origine du déclenchement de processus dirigés contre des cellules normales du « soi », engendrant ainsi des réactions auto-immunes sévères. L'enjeu majeur de l'immunothérapie en cancérologie est donc d'éliminer les cellules anormales sans affecter les cellules saines.

Depuis les travaux pionniers de S. Rosenberg, au National Cancer Institute $(\mathrm{NCl})$, on sait qu'une thérapie cellulaire adoptive (c'est-à-dire par transfert de cellules immunocompétentes et donc possiblement actives contre des cellules cancéreuses) peut conduire à des réponses cliniques complètes dans le cas du mélanome métastatique [8]. Cette thérapie est efficace si elle est réalisée par transfert de cellules effectrices de l'immunité adaptative ou innée en nombre suffisant et si celles-ci reconnaissent spécifiquement les cellules tumorales grâce à des molécules ayant une forte affinité pour une cible moléculaire exprimée à la surface de ces dernières [9] $(\rightarrow)$.

$(\rightarrow)$ Voir la Synthèse de V. Catros et al., $\mathrm{m} / \mathrm{s}$ $n^{\circ} 2$, février 2010, page 185

Leur efficacité est d'autant plus grande quand ces cellules combinent un stade de différenciation à fort potentiel cytotoxique avec une capacité à poursuivre leur expansion in vivo après injection. Toutes ces propriétés sont remplies par les lymphocytes infiltrant la tumeur (TIL) lorsqu'ils sont spécifiques des antigènes associés aux tumeurs (TAA : tumor-associated antigen). Optimiser ex vivo la préparation de tels lymphocytes pour qu'ils remplissent les fonctions attendues contre les cellules tumorales reste cependant encore un véritable défi [10].

Au cours de la réponse lymphocytaire T spécifique, la reconnaissance du TAA repose sur le récepteur que ces cellules expriment, le TcR ( $T$ cell receptor). L'affinité pour des TAA des TcR exprimés par les lymphocytes $T$ est faible par rapport à celle des TcR spécifiques d'antigènes viraux par exemple. Ceci explique les résultats spectaculaires qui sont obtenus avec un transfert adoptif de TIL à des patients qui présentent des tumeurs viro-induites exprimant de tels antigènes [10]. Ces observations ont conduit au développement de structures moléculaires présentant de meilleures fonctionnalités soit en améliorant la qualité du TcR utilisé, soit en permettant au lymphocyte $T$ de lier sa cible en utilisant des constructions améliorant l'affinité du TcR pour son ligand. II s'agit là d'un domaine de la biologie de synthèse qui relève à la fois de la thérapie cellulaire et de l'ingénierie moléculaire [11].

Dans ce type d'approches, deux axes de recherche sont menés de front, souvent par les mêmes équipes afin d'améliorer ex vivo la qualité des lymphocytes qui seront transférés. Le premier consiste à transférer dans les lymphocytes cytotoxiques un TcR modifié afin qu'il présente une très forte affinité pour l'antigène tumoral : ce sont les haTcRT (high affinity TcR-engineered $T$ cell). Comme les TIL, ces cellules reconnaissent les TAA à la surface des cellules tumorales dans le contexte des molécules du complexe majeur d'histocompatibilité (CMH) de classe I ou II (Figure 2). La seconde méthode consiste à faire exprimer par le lymphocyte T du patient un récepteur particulier dérivé d'un anticorps spécifique de l'antigène tumoral (permettant sa reconnaissance) fusionné à une région transmembranaire et à une ou plusieurs unités de transduction du signal permettant l'activation de ce lymphocyte $T$ : ces CAR (chimeric antigen receptor engineered T cells) créent alors une synapse immunologique qui sera spécifique des cellules tumorales en dehors de toute implication des molécules du CMH. Les CAR combinent ainsi reconnaissance spécifique de l'antigène tumoral par un anticorps et capacité du lymphocyte $T$ à tuer les cellules exprimant l'antigène cible. Ces deux méthodes d'ingénierie cellulaire ont été développées dans un objectif d'utilisation clinique. 


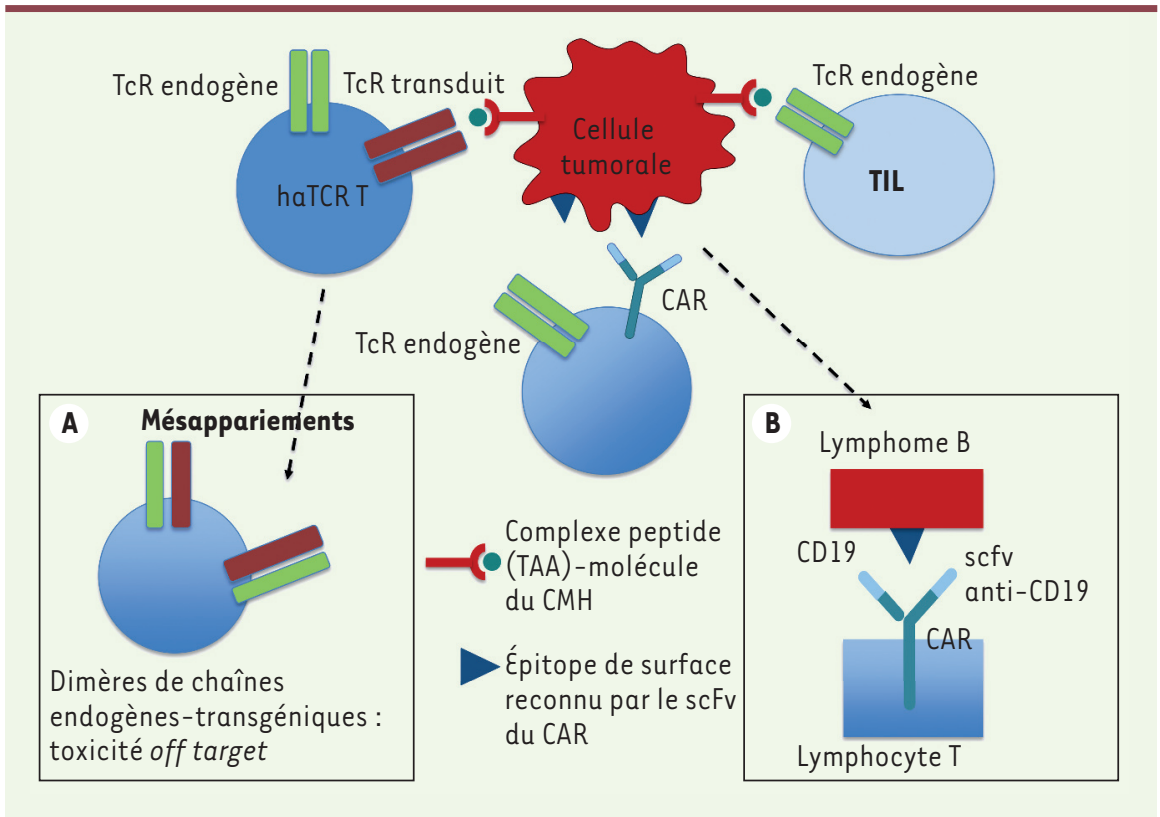

Figure 2. Lymphocytes T modifiés par transfert de gène. Les lymphocytes T peuvent être modifiés par ingénierie cellulaire pour améliorer leur affinité pour la cellule tumorale ciblée. Les TIL et les haTcRT reconnaissent les peptides dérivés des TAA présentés par les cellules tumorales associés à des molécules de classe I du complexe majeur d'histocompatibilité (CMH). Les cellules CAR reconnaissent un antigène présenté par la cellule tumorale par la portion scFv de leur récepteur chimérique, hors contexte CMH. A. Un des risques des haTcRT est la formation de dimères avec les chaînes des TcR endogènes. Ces dimères peuvent avoir une spécificité pour d'autres antigènes et générer de la off toxicité. B. Dans le cas du CAR anti-CD19, la molécule CD19 ciblée est exprimée à la surface des cellules de lymphome $B$.

\section{Construction moléculaire des CAR}

Les récepteurs chimériques des CAR sont des polypeptides qui sont constitués de plusieurs modules distincts : un module extracellulaire qui se liera à la cible, constitué d'un fragment simple chaîne d'anticorps (scFv), fait d'un domaine VH (domaine variable de la chaîne lourde de l'anticorps) relié par un court polypeptide flexible au domaine VL (domaine variable de la chaîne légère) du même anticorps; un module d'ancrage transmembranaire; et un ou plusieurs modules intracellulaires qui sont impliqués dans la signalisation lymphocytaire T (Figure 3). Les lymphocytes $T$ du patient sont isolés par leukaphérèse, une méthode de tri spécifique des cellules sanguines. Ils sont ensuite modifiés génétiquement à l'aide de vecteurs lentiviraux ou $\gamma$-rétroviraux ${ }^{2}$ codant la molécule complète du CAR considéré. Un ratio équilibré de lymphocytes T CD8 et de lymphocytes T CD4 dans les cellules isolées des patients permet une bonne faisabilité du protocole pour plus de $90 \%$ d'entre eux, même pour ceux qui présentent une lymphopénie sévère [12]. L'utilisation de sous-populations purifiées de lymphocytes T naïfs pourrait améliorer à terme la fonctionnalité des cellules et est actuellement expérimentée. Les rétrovirus de grade GMP ${ }^{3}$ qui sont utilisés n'expriment plus les gènes qui leur sont essentiels : gag, pol, et env. Ceci permet la réplication du virus et sa production sous la forme de particules qui pénètreront dans la cellule sans qu'il y ait production efficace du virus; ils sont donc dits non réplicatifs. Ils ne doivent pas infecter d'autres cellules, non ciblées. Le risque de voir resurgir des virus réplicatifs, bien que très faible, fait l'objet de contrôles stricts imposés aux États-Unis pendant 15 ans par la Food and drug administration (FDA) chez les patients qui ont été traités [13]. Certaines équipes privilégient des méthodes de transfert de

${ }^{2}$ Les $\gamma$-rétrovirus comprennent entre autres, le MLV (virus de la leucémie murine ou virus de Moloney) et sont utilisés pour le transfert de gènes.

${ }^{3}$ Good Manufacturing Practices. Ce sont des virus dont la production est strictement contrôlée. gènes en l'absence de virus, telles que l'électroporation ou l'utilisation de transposons pour, d'une part, restreindre les effets adverses et l'antigénicité potentielle des vecteurs rétroviraux, et, d'autre part, simplifier les procédures de préparation cellulaire.

Les CAR anti-CD19 (les premiers à avoir été utilisés avec succès pour le traitement de patients ayant une leucémie aiguë lymphoblastique [LAL]) sont des cellules qui expriment de façon constitutive à leur surface des récepteurs chimériques résultant de constructions constituées de la séquence codant un scFv dérivé d'un anticorps anti-CD19 de forte affinité. L'espaceur utilisé entre les domaines VH et VL est la plupart du temps un motif Gly-Gly-GlyGly-Ser répété deux ou trois fois. Le choix de l'anticorps anti-CD19 repose sur le fait que cette molécule est spécifiquement exprimée à la surface des lymphocytes B normaux et tumoraux. La flexibilité du scFv anti-CD 19 est assurée par une région charnière qui le sépare de la membrane cellulaire. La partie transmembranaire de la construction est constituée de la séquence transmembranaire de molécules impliquées dans la fonction des lymphocytes T. Enfin, la région intracellulaire contient un ou deux modules de transduction du signal, dérivés de molécules de signalisation qui sont des co-récepteurs activateurs des lymphocytes $T$.

Les deux premières générations de CAR utilisaient la région transmembranaire de CD8 ou de CD4 et le module de transduction inséré dans la partie intracellulaire était un des domaines activateurs de molécules de co-stimulation des lymphocytes T (CD28 ou 4-1BB [CD137] ou OX40 [CD134]). Les CAR de troisième génération combinent désormais au moins deux domaines de molécules 


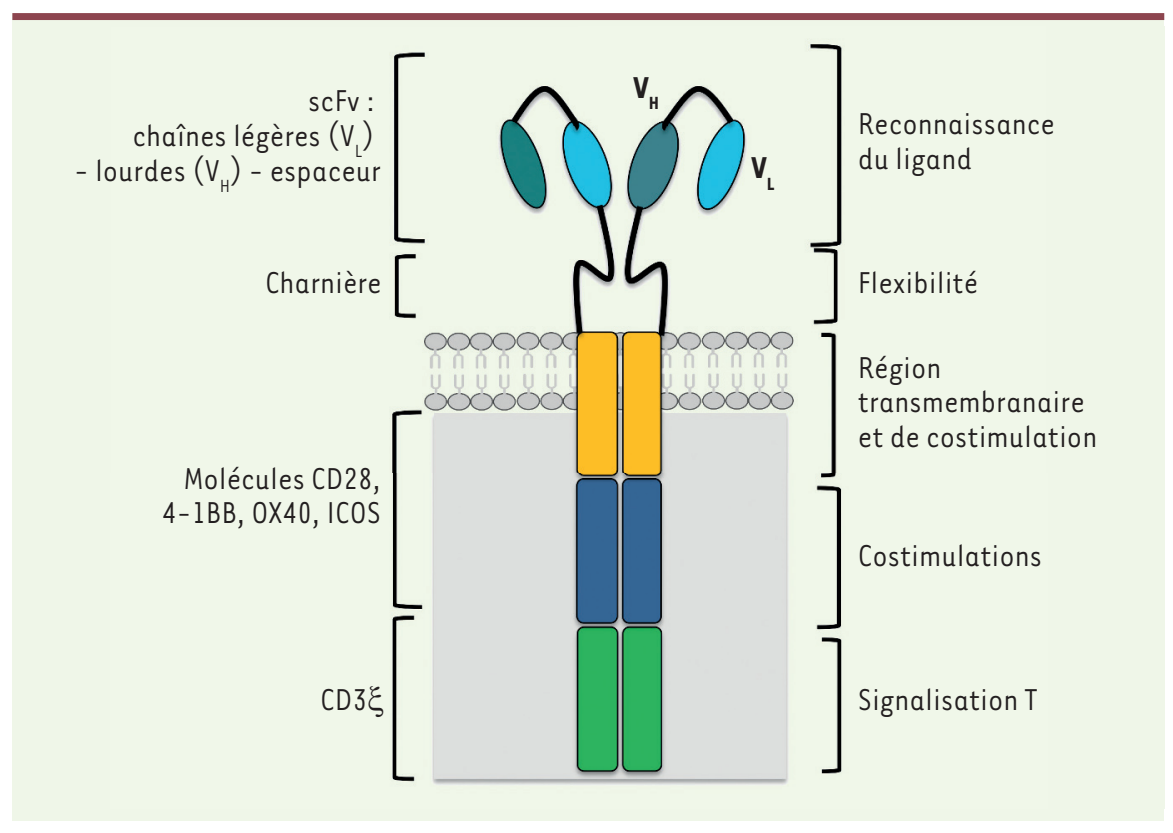

Figure 3. Récepteur chimérique d'antigène (CAR). Le prototype des CAR comprend 3 domaines canoniques: un pour la liaison à l'antigène (non restreinte par les molécules du complexe majeur d'histocompatibilité [CMH]), un pour l'activation du lymphocyte $T$ (en général, le domaine intracellulaire de la chaîne $\mathrm{CD} 3 \zeta$ ) et un domaine de co-stimulation, comme par exemple le domaine intracellulaire de CD28. Dans les CAR de troisième génération des domaines intracellulaires de CD137/4-1BB, CD134/0X40 ou ICOS sont ajoutés à la construction.

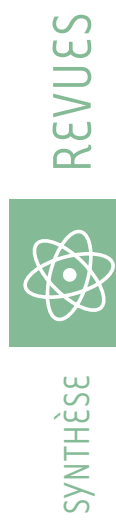

de co-stimulation comprenant l'un des domaines cités ci-dessus. Ces séquences de co-stimulation sont associées au domaine de signalisation de la chaîne $\zeta$ du complexe $\operatorname{CD} 3(\operatorname{CD} 3 \zeta)$ du récepteur T de l'antigène (TcR) à l'extrémité C-terminale du CAR produit. Toutes ces constructions conduisent à l'expression de récepteurs recombinants permettant au lymphocyte T de s'activer lorsqu'il reconnaît sa cible antigénique et de lyser les cellules qui expriment cette cible.

Pour obtenir les CAR, les lymphocytes T sont activés par un cocktail d'anticorps monoclonaux anti-CD3 (comme OKT3) et anti-CD28 couplés à des billes puis par de l'IL-2 et infectés par un virus recombinant codant le récepteur chimérique. Les cellules infectées sont alors cultivées in vitro en présence d'IL-2 ce qui permet de les faire proliférer jusqu'à l'obtention du nombre de cellules nécessaires à leur utilisation par voie intraveineuse en clinique, soit de 0,2 à $5 \times 10^{6} \mathrm{CAR}^{+}$viables $/ \mathrm{kg}$ pour les patients de moins de $50 \mathrm{~kg}$ et de 0,1 à $2,5 \times 10^{8} \mathrm{CAR}^{+}$cellules T viables pour les patients de plus de $50 \mathrm{~kg}$ dans les LAL de l'enfant et du jeune adulte et de 0,6 à $6 \times 10^{8}$ (KYMRIAHTM) ou $2 \times 10^{6} / \mathrm{kg}$ avec un maximum de $2 \times 10^{8} / \mathrm{kg}\left(\right.$ YESCARTA $\left.^{\text {TM }}\right)$ cellules T CAR ${ }^{+}$viables pour les patients adultes présentant un lymphome diffus à grandes cellules $B$ (LDGCB) réfractaire ou en rechute.

\section{L'avancée des cellules CAR en clinique}

Les premiers CAR ont été conçus par Z. Eshhar en 1989 ; il les avait alors appelés T-bodies [14]. Leur évaluation dans des études précliniques réalisées dès 1990 avait néanmoins révélé une toxicité sévère ainsi qu'une incapacité à maintenir leur activité cytotoxique après infusion du fait du type de constructions alors utilisées [15]. En 2003, des essais menés in vitro et in vivo ont en revanche montré le puissant potentiel thérapeutique de CAR anti-CD19 [16]. Dès 2008, l'équipe de M. Brenner, du Baylor College of Medicine à Houston, avait pu obtenir, dans un essai incluant 11 patients, une réponse clinique complète chez une petite fille de 4 ans atteinte d'un neuroblastome en utilisant des CAR spécifiques du GD2 (disialoganglioside) [17]. Mais c'est seulement en 2010 que des cas de régression tumorale spectaculaires ont été rapportés dans le cadre d'essais de phase I, avec des CAR anti-CD19 de deuxième génération pour des lymphomes folliculaires B [18], des leucémies lymphoïdes chroniques (LLC) [19] ou aiguës (LAL) [20]. Les injections de cellules modifiées étaient précédées de chimiothérapies lymphoablatives, à base de fludarabine et de cyclophosphamide afin d'éliminer les lymphocytes $T$ régulateurs (Treg) chez les patients. C'est à l'occasion de ces essais que les équipes de C. June, de l'Université de Pennsylvanie (UPENN) à Philadelphie, et de M. Sadelain au Memorial Sloan-Kettering Cancer Center (MSKCC) à New York, ont décrit la fonte de masses tumorales volumineuses en quelques jours. Citons ainsi l'exemple d'un patient atteint de leucémie lymphoïde chronique $B$ (LLC-B) CD19+, en rechute de sa maladie et réfractaire à tout traitement [19]. Son inclusion dans un essai de phase I lui a permis de recevoir $3 \times 10^{8}$ cellules modifiées, dont seulement $5 \%$ étaient des CAR anti-CD19 de deuxième génération. Les adénopathies qu'il présentait et qui étaient suivies par des examens par scanner de façon répétée, ont alors disparu en 28 jours. Le caryotype de ses cellules s'est normalisé et les cellules B anormales ont disparu de la moelle osseuse, parallèlement à la détection d'une réponse immunitaire spécifique. Les cellules transférées ont persisté durant 6 mois dans le sang et la moelle osseuse.

En 2013, un autre essai pionnier portant sur 5 patients, qui a été validé un an après sur 16 autres patients, 
a confirmé la réduction rapide de masses ganglionnaires volumineuses chez ces patients atteints de LAL réfractaires à la chimiothérapie. Les CAR utilisés exprimaient un récepteur chimique «19-28z » constitué d'un scFv anti-CD19 et du domaine d'activation intracellulaire de CD28 [20]. Plus de $80 \%$ de réponses complètes ont été observées dans cet essai et les patients sont devenus éligibles à une greffe de cellules souches hématopoïétiques [21]. Cet essai a par ailleurs établi que les réponses immunitaires des patients traités étaient corrélées à leurs réponses cliniques: les CAR s'activent en reconnaissant $\mathrm{CD} 19$ et tuent leur cible. Néanmoins, l'efficacité du traitement s'est traduite par une association à un syndrome rapide de production massive de cytokines (CRS, pour cytokine release syndrome ou orage cytokinique). Les clones de cellules modifiées ont persisté plusieurs années et ont permis un contrôle de la maladie résiduelle. Cet avantage s'est parfois transformé en effet indésirable car les CAR peuvent aussi cibler les lymphocytes B CD19+ normaux, ce qui a conduit à des lymphopénies ou à des aplasies médullaires sévères.

Toutes ces toxicités justifient l'hospitalisation en soins intensifs des patients traités. Celles-ci sont désormais contrôlées en partie par des corticoïdes. Des rémissions obtenues après un nombre limité d'injections d'un faible nombre de cellules activées sont maintenant observées après plus de 5 ans [22].

Plusieurs sociétés pharmaceutiques se sont engagées dans le développement industriel des CAR [23] (Figure 5) $(\rightarrow$ ). En 2017, la société Novartis, qui travaille en collaboration avec l'université de Pennsylvanie (UPENN), a obtenu l'approbation par la FDA du premier médicament CAR-T cells : il s'agit du

\section{$\rightarrow$ Voir la Chronique génomique de $B$. Jordan, $m / s n^{\circ} 11$, novembre 2017, page 1003} KYMRIAHTM (tisagenlecleucel), un CAR anti-CD19 destiné à des enfants en échec thérapeutique d'une LAL. L'autorisation a été obtenue bien avant la fin de l'essai clinique programmé pour 2024, 83 \% de rémissions ayant été observées dès les 70 premiers patients inclus au cours des trois premiers mois de l'essai.

La logistique entourant les 20 centres de prélèvements, qui assurent la cytaphérèse (isolement des lymphocytes des patients), et le laboratoire central, qui assure la préparation des CAR (modification génique et multiplication des cellules modifiées), sont gérés par Novartis. La gestion rapide de la toxicité de ces traitements est très standardisée. Ce médicament est en essai clinique pour d'autres types de tumeurs.
La FDA a également autorisé un autre CAR anti-CD19, le YESCARTA'M (axicabtagene ciloleucel, commercialisé par la société Gilead) pour le traitement de lymphomes non hodgkiniens (LNH) agressifs de l'adulte 4 . L'ingénierie de ce CAR résulte d'un partenariat entre le National Cancer Institute $(\mathrm{NCl})$ américain et la société Kite Pharma propriétaire du brevet et récemment rachetée par Gilead [23]. Le YESCARTATM a obtenu en juillet 2018 I'autorisation temporaire d'utilisation et d'essai en France pour le traitement de patients adultes atteints de lymphome non hodgkinien agressif ou indolent réfractaire, de lymphome des cellules du manteau adulte, de LAL de l'adulte et de l'enfant (essais ZUMA 1-5). Ces essais sont multicentriques et sont réalisés dans plusieurs hôpitaux français. Le coût actuel de ces traitements dépasse 300000 euros par patient.

La société JUNO Therapeutics a été fondée en 2013 par des chercheurs, pionniers des CAR au Memorial Sloan-Kettering Cancer Center à New York et au Fred Hutchinson Cancer Center de Seattle (États-Unis). Elle est engagée dans des essais de phase I et II multicentriques pour évaluer les CAR 19-28z dans des lymphomes non hodgkiniens, mais aussi les CAR antiWTl (Wilms Tumor 1) dans la leucémie aiguë myéloïde

\footnotetext{
${ }^{4}$ Les lymphomes diffus à grandes cellules B sont les formes les plus communes des lymphomes non hodgkiniens (LNH) à cellules B. Les lymphomes folliculaires, ceux de la zone marginale, et ceux des cellules du manteau sont aussi des LNH à cellules B.
} 
(LAM), anti-CD22 dans des lymphomes non hodgkiniens et les LAL et des multi-CAR en combinaison avec des anticorps thérapeutiques comme les anti-points de contrôle inhibiteurs (ICP) notamment. Les multi-CAR peuvent être des mélanges de plusieurs CAR présentant des spécificités différentes ou des CAR construits pour reconnaître plusieurs cibles [9].

La société Cellectis développe, elle, des CAR allogéniques ${ }^{5}$, afin d'éviter le besoin de lymphocytes autologues (isolés du patient luimême afin d'éviter tout problème de rejet ou de réaction du greffon contre l'hôte - GvHD). Elle met au point des CAR « universels », n'exprimant plus de récepteur $T$ de l'antigène, qui pourraient être stockés et utilisés à la demande (therapy off the shelf). Les réactions immunitaires induites par ces cellules freinent cependant leur avancée en clinique. D'autres CAR sont en développement dans les tumeurs hématologiques. Ils ciblent notamment CD138 ou la molécule $B$-cell maturation antigen (BCMA) dans le myélome, CD33 et CD123 dans la leucémie myéloïde chronique (LMC), et CD22 dans les LAL [10]. Pour chacun de ces ligands, la conception et l'optimisation du récepteur chimérique a ses particularités.

\section{Efficacité et toxicité : ce qui ne tue pas renforce}

En 2018, l'efficacité des CAR anti-CD19 a été reconnue. Un taux de $90 \%$ de réponses a été en effet rapporté pour les patients en rechute de LAL à cellules $B$ et de plus de $60 \%$ pour les patients en rechute de lymphomes non hodgkiniens à des stades avancés [24].

Les CAR peuvent aussi induire des évènements indésirables graves qui engagent le pronostic vital du patient. Lorsque les CAR reconnaissent leur cible, ils libèrent des quantités massives de cytokines inflammatoires, comme le TNF- $\alpha$ (tumor-necrosis factor alpha), I'IFN- $\gamma$ (interféron gamma) et de multiples autres cytokines, comme les IL-1, $-2,-6$ et -8 qui sont à l'origine du recrutement en cascade de différentes cellules de l'immunité innée et adaptative et qui amplifient à leur tour la réponse de ces cellules vis-à-vis de l'hôte. Les symptômes les plus fréquents sont une fièvre, une hypotension, une hypoxie, une tachycardie et des frissons. Mais une tempête cytokinique excessive peut conduire à une hyperperméabilité capillaire qui peut être à l'origine d'un œdème cérébral, d'une insuffisance cardiaque ou d'une atteinte rénale aiguë. L'emballement de la réponse immunitaire peut être provoquée par l'activation de cellules immunitaires par de faibles niveaux d'antigènes reconnus par les CAR exprimés sur d'autres tissus : la toxicité est alors dite « on target ». L'orage cytokinique peut aussi résulter de la reconnaissance de structures présentant un mimétisme antigénique avec l'antigène tumoral, par réaction croisée: la toxicité est alors dite «off target ». La lyse massive et rapide des cellules tumorales peut donc également conduire à une toxicité.

L'analyse des biomarqueurs produits au cours des phases aiguës de la réponse a permis de trouver des solutions thérapeutiques. Ainsi, le tocilizumab (RoActemra ${ }^{T M}$ ), un anticorps inhibiteur du récepteur

${ }^{5}$ Les lymphocytes modifiés qui seront injectés ne proviennent pas du patient. de I'IL-6, est maintenant inscrit dans l'arsenal thérapeutique des essais cliniques utilisant des CAR et susceptibles de s'accompagner d'une violente réponse inflammatoire. Son utilisation est un bel exemple de sérendipité [25].

Les chimiothérapies lymphoablatives qui sont réalisées avant l'infusion des CAR chez les patients sont aussi responsables de neurotoxicité : leur choix et leurs doses sont en cours de standardisation. Aujourd'hui, une meilleure maîtrise des conditionnements chimiothérapeutiques, des sous-populations cellulaires soumises à l'ingénierie, des plateformes de préparation, des doses injectées, et l'administration préventive de corticostéroïdes et d'anticorps anticytokines, permettent de progresser dans les essais thérapeutiques des CAR [12, 26] (Figure 5).

\section{Application des CAR aux tumeurs solides}

Les résultats obtenus dans des tumeurs hématologiques avec les CAR ont incité les équipes à poursuivre l'ingénierie de ces cellules pour des applications aux tumeurs solides [27]. Les anticorps choisis sont peu nombreux : peu de protéines de surface strictement spécifiques de ces tissus tumoraux existent [7]. Des peptides antigéniques peuvent être présentés dans certains types histologiques de tumeur, mais ils sont souvent partagés par des tissus sains essentiels. C'est pour cette raison que des peptides de TAA, comme MART-1 (melanomaassociated antigen recognized by $T$ cells 1 ), NY-ESO-1 (New York esophageal squamous cell carcinoma 1), gp100 et CAPl (cyclase-associated protein 1) (issu de l'antigène carcinoembryonnaire : $A C \varepsilon$ ), sont dits « associés aux tumeurs » ou « embryonnaires » [7]. L'optimisation de la spécificité de tels CAR est donc complexe.

Le tout premier essai clinique mené en 2010 avec des CAR construits avec le scFv de l'anticorps ciblant HER2 (human epidermal growth factor receptor 2) a révélé une toxicité pulmonaire fulgurante et létale, alors même que la toxicité de l'Herceptin ${ }^{T M}$, un anticorps utilisé dans le cancer du sein métastatique HER2+ (avec surexpression de ce récepteur) est modérée [25]. Le variant 3 d'un récepteur de la même famille (EGFRvIII) est une cible intéressante : il est exprimé spécifiquement par certaines tumeurs cérébrales [28]. Une réponse complète et de longue durée a pu être observée avec des CAR spécifiques de ce récepteur. Ils sont maintenant en évaluation dans plusieurs centres.

Des CAR de deuxième ou de troisième génération ciblant HER2, la mésothéline, l'ACE mais aussi le GD2-0-acétylé (forme 0 -acétylée de GD2), MUCl (mucine 1), cMET 


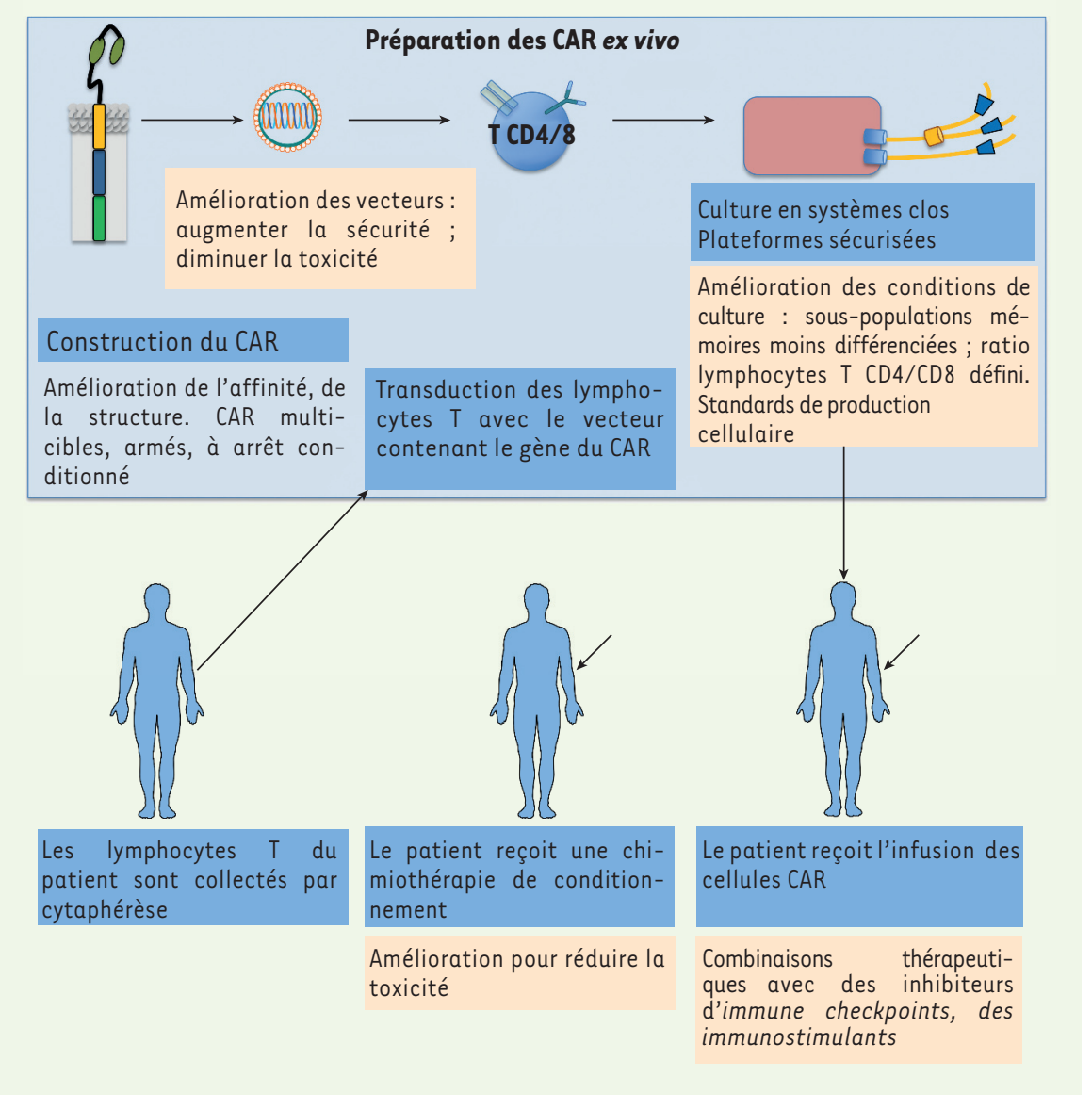

Figure 5. Grandes étapes et améliorations en cours pour les thérapies cellulaires par les CAR-T cells. Les lymphocytes T autologues sont obtenus à partir d'un prélèvement de sang ou une cytaphérèse. Les cellules sont transduites ex vivo avec les gènes de CAR à l'aide de gammarétrovirus, lentivirus ou par une méthode de transfert non viral. L'expansion ex vivo est réalisée dans des plateformes contrôlées pour tous les aspects de sécurité et de reproductibilité. Les cellules sont ré-administrées au patient par voie intraveineuse après un conditionnement chimiothérapeutique. Les CAR circulent dans l'organisme jusqu'à reconnaître leur antigène spécifique, s'activer et détruire les cellules tumorales. Les prochaines générations de CAR bénéficieront d'améliorations à toutes les étapes: la conception du CAR, le phénotype des cellules transduites, le contrôle de la fonction du CAR, leur combinaison avec d'autres approches thérapeutiques.

(tyrosine-protein kinase Met), ROR1 et ROR2 (receptor tyrosine kinaselike orphan receptor 1 et 2), CD133 (prominin-1), CD171 (LICAM : LI cell adhesion molecule) sont désormais en évaluation clinique de phase I/II aux États-Unis, en Chine et en Europe pour différents types de tumeurs $[25,26]$.

En parallèle, une nouvelle voie de ciblage des tumeurs solides s'ouvre en raison de leurs particularités métaboliques. Les mécanismes de 0 -glycosylation souvent anormaux dans les tumeurs sont à l'origine de l'expression d'antigènes pour lesquels des CAR peuvent être conçus: les $5 \varepsilon 5-C A R$ ciblent des formes glycosylées particulières de la mucine MUCl ( $T n-M U C l$ ) qui sont présentes dans les adénocarcinomes pancréatiques, coliques et de l'ovaire, des tumeurs contre lesquelles des essais sont menés actuellement [29]. Des CAR anti-VEGF-R2, le récepteur du VEGF (vascular endothelial growth factor) permettent également le ciblage du microenvironnement tumoral [25].

\section{Les obstacles à surmonter pour accéder aux tumeurs solides}

D'autres raisons que leur spécificité antigénique expliquent les échecs observés avec les premières générations de CAR dirigés contre des tumeurs solides et les difficultés rencontrées. Contrairement aux cellules sanguines anormales, les cellules de tumeur solide sont pro- tégées par de multiples barrières provenant de leur microenvironnement. La nature fibreuse de la matrice extracellulaire et les anomalies des cellules endothéliales sont ainsi des barrières physiques à la migration des CAR qui, provenant du sang, doivent rejoindre le site tumoral et pénétrer au sein du stroma tumoral. Le $\mathrm{pH}$ acide, l'hypoxie et le très faible apport en nutriments (glucose, glutamine, L-arginine) constituent également un environnement hostile à la survie et à la multiplication in situ de ces lymphocytes thérapeutiques [30]. Le métabolisme glycolytique tumoral et l'inflammation chronique qui s'installent au sein des tumeurs solides conduisent de plus à de fortes concentrations locales en molécules et cytokines immuno-régulatrices. Cet état est favorable au recrutement de lymphocytes $T$ régulateurs (Treg), de cellules suppressives myéloïdes (MDSC), de macrophages immunosuppresseurs (TAM) et à l'expression de récepteurs inhibiteurs qui s'opposent à l'activité des CAR. Ces écueils ne peuvent être surmontés que par le développement de constructions moléculaires plus élaborées, relevant parfois de la biologie synthétique combinatoire, et dites de « prochaine génération » (next generation CAR-T cells). 


\section{Les améliorations attendues des next generation CAR-T cells}

Les efforts d'ingénierie qui visent à optimiser les résultats cliniques en minimisant les effets indésirables portent sur la spécificité, la survie, l'adressage des CAR à la tumeur et l'arrêt de leur activité [11, 27] (Figure 5). La longueur de la construction, la taille et la flexibilité de la région charnière du CAR sont des caractéristiques qui peuvent être optimisées, mais chaque antigène tumoral est particulier [11]. Par exemple, les propriétés de l'espaceur situé entre la partie scFv et la région transmembranaire, qui améliorent l'affinité pour l'épitope reconnu par les CAR spécifiques de RORl, en essai dans le cancer du sein, diffèrent de celles d'autres CAR [31].

Les CAR n'ont pas la même fonctionnalité selon le module de co-stimulation choisi pour leur construction [27]. Les BBzCAR constitués d'un module intracellulaire dérivé de 4-1BB ont ainsi une biogenèse mitochondriale accrue qui est favorable à leur différenciation en lymphocytes T centraux mémoires; les 28zetaCAR qui contiennent un module intracellulaire dérivé de CD28 sont polarisés vers un métabolisme glycolytique et une différenciation en lymphocytes T effecteurs mémoires [32]. Le choix du module intracellulaire de la construction conditionne ainsi la capacité de division ou d'épuisement des CAR après leur injection. Des optimisations sont expérimentées pour des CAR de troisième génération en ajoutant un deuxième module de costimulation, comme ICOS (inducible costimulator) [33], ou en remplaçant le module dérivé

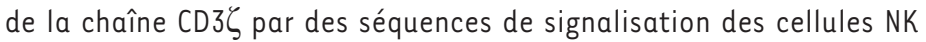
(natural killer) [25]. Les CAR de quatrième génération présentent une séquence permettant la production de cytokines: la reconnaissance de la cible entraîne ainsi l'activation d'un facteur de transcription permettant l'expression d'IL-12 ou d'IL-18 [34].

On assiste aujourd'hui à l'arrivée des tous premiers multi-CAR en essai clinique $[11,27,35]$. Ils expriment plusieurs récepteurs chimériques constitués de scFv spécifiques de différentes cibles antigéniques. Ces molécules chimériques différentes peuvent être transduites dans la même cellule, c'est la logique du «AND ». Elles peuvent également être introduites dans des cellules différentes qui seront combinées au moment du traitement, c'est la logique du «OR ». Ces combinaisons permettent d'augmenter la reconnaissance spécifique des CAR et de réduire leur toxicité off target en abaissant les doses de chacune des deux populations de CAR. Ces stratégies sont en préparation pour des essais de traitement des glioblastomes dans lesquels des CAR anti-EGFRvIII, -HER2, -IL13Ra2 ont déjà fait, séparément, l'objet d'essais cliniques et pourraient être combinés à des anticorps thérapeutiques anti-PD-1 [36] Les équipes pionnières dans le domaine des CAR cherchent aussi de nouvelles voies d'amélioration à partir de leurs observations cliniques. Elles tentent en effet de comprendre pourquoi certains patients ont répondu avec des guérisons spectaculaires. Le cas du patient $n^{\circ} 10$ de l'essai CTL019 mené à l'université de Pennsylvanie est très informatif

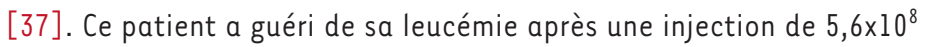
lymphocytes modifiés; I'analyse de ses lymphocytes circulants, 2 mois après l'infusion, a révélé que plus de $98 \%$ de son répertoire était clonal. Le transgène s'était inséré dans un locus impliqué dans le mécanisme d'activation des lymphocytes $T$ : c'est ce clone qui a guéri le patient ! L'insertion du transgène dans un «bon » locus par des méthodes CRISP/Cas9 sous I'influence d'un promoteur adapté est ainsi un objectif poursuivi par plusieurs équipes [24]. Une insertion dirigée influencerait non seulement la puissance du CAR, mais aussi sa persistance sans phénomène d'épuisement [24]. Dans ce cas, les doses de cellules injectées pourraient être réduites, ce qui permettrait de diminuer les effets adverses que l'on observe.

Les prochaines générations de CAR devront éviter les effets délétères d'une persistance trop importante des clones modifiés. Des systèmes d'arrêt d'activité, comme l'expression conjointe d'un CAR inhibiteur (iCAR) reconnaissant un autre antigène exprimé par les cellules saines avec des modules de transduction inhibiteurs empruntés aux molécules PD-1 pourraient permettre d'atteindre cet objectif [12, 24]. L'activité du CAR peut aussi être modulée en ajoutant un gène de délivrance d'un signal apoptotique par induction de la caspase 9 [24].

Les essais cliniques actuels sont réalisés avec des cellules autologues pour éviter les risques immunologiques et de GvHD, mais des transferts de CAR dans des cellules souches précurseurs de lymphocytes $T$ modifiés pourraient permettre d'utiliser des cellules allogéniques et de développer des CAR « off the shelf » [12, 38] (Figure 5).

Plus de 40 essais cliniques de CAR dans des tumeurs solides sont actuellement référencés sur le site https:// clinicaltrials.gov/ des National Institutes of Health. Ils combinent parfois l'inhibition de la tolérance par des ICP et le ciblage de plusieurs récepteurs selon les concepts des CAR de «prochaine génération ». Des essais sont en cours en Chine pour des stades avancés de tumeurs solides avec des anticorps anti-CTLA-4 et anti-PD- 1 et des CAR spécifiques de la mucine $M U C l$, du récepteur EGFR et de la mésothéline.

\section{Le transfert de TcR : haTcRT (TcR-engineered $T$ cell)}

Depuis les essais de thérapie adoptive par des TIL dans le mélanome, il a été montré que des réponses complètes et durables peuvent survenir lorsque des lymphocytes T CD8 spécifiques des TAA sont injectés aux patients. Leur efficacité est corrélée à l'affinité du TcR qu'elles expriment. Lorsque ces TIL spécifiques peuvent être triés et amplifiés, leur pouvoir thérapeutique peut être amélioré si l'injection est précédée d'un conditionnement lymphoablatif [8].

C'est de ces constats, ainsi que des difficultés rencontrées pour l'expansion des lymphocytes de patients atteints de cancer [39], qu'a émergé l'idée de modifier des lymphocytes $T$ afin de leur faire exprimer un TcR qui soit spécifique de TAA avec une affinité optimale : les haTcRT. 
Le transfert de chaînes de TcR $\alpha$ et $\beta$ dans des lymphocytes T primaires est une technologie maîtrisée depuis 20 ans, mais elle génère des cellules qui continuent d'exprimer leur TcR endogène et la reconnaissance de l'antigène ciblé nécessite sa présentation par les molécules du $\mathrm{CMH}$ (Figure 2).

En 2006, R. Morgan, du laboratoire de S. Rosenberg, a publié les premiers résultats cliniques obtenus avec des lymphocytes T modifiés pour reconnaître MélanA (ou MART-1, melanoma-associated antigen recognized by $T$ cells) dans le mélanome métastatique. Deux des 17 patients qui avaient été traités ont montré une réponse, mais les résultats n'étaient pas supérieurs à ceux obtenus avec des TIL utilisés dans ce même essai.

Des essais cliniques ont ensuite été menés dans différents contextes de $\mathrm{CMH}$, avec des TcR d'avidité et d'affinité optimisées. Dans le myélome multiple, $80 \%$ de réponses cliniques ont été obtenues en ciblant NY-ESO-1, sans toxicité. Par contre, d'importantes toxicités cutanées, oculaires, auditives et de dépigmentation sont survenues dans la majorité des autres essais [25]. Dans les essais ciblant MAGEA3 (melanoma-associated antigen-A3), des toxicités sévères sont apparues entraînant parfois le décès du patient moins de 7 jours après I'infusion des lymphocytes T. Actuellement, des essais de haTcRT spécifiques d'antigènes d'HBV (virus de l'hépatite $B$ ), d'HPV E6 (human papillomavirus $\varepsilon 6$ ), de MAGE-A3, MAGE-A4, NY-ESO-1, ou WTl, sont en cours pour le traitement de tumeurs solides ou hématologiques [25]. Les néoantigènes résultant d'instabilités des microsatellites ou d'origine virale sont également des cibles prometteuses. La spécificité de ces antigènes tumoraux est importante et leur tolérance est limitée. Néanmoins, leur développement ne pourra être réalisé que dans le cadre de thérapies personnalisées après le séquençage de l'exome de leur tumeur.

\section{GLOSSAIRE}

CAR : chimeric antigen receptor modified T cells

CRS : syndrome de libération de cytokines ou orage cytokinique

Fab: domaine d'une immunoglobuline responsable de la reconnaissance de l'antigène par l'anticorps. Le fragment Fab d'un anticorps a une affinité pour l'antigène proche de celle de l'anticorps entier. II est formé de la chaîne légère entière $\left(V_{L}+C_{L}\right)$ et d'une partie de la chaîne lourde $\left(\mathrm{V}_{\mathrm{H}}+\mathrm{C}_{\mathrm{H}}\right)$. Il est monovalent

GuHD : graft-versus-host disease

haTcRT : high affinity TcR-engineered T cell

ICP : Immune checkpoints inhibitor

LA : leucémie aiguë

LAL : leucémie aiguë lymphoblastique

LAM : leucémie aiguë myéloblastique

LLC : leucémie lymphoïde chronique

LMC : leucémie myéloïde chronique

LNH : lymphome non hodgkinien

TAA : tumor-associated antigen

TAM : tumor-associated macrophage

TIL : tumor-infiltrating lymphocyte
L'analyse des effets toxiques observés avec les haTcRT révèle qu'ils résultent de la reconnaissance, par ces cellules modifiées, d'antigènes qui sont exprimés par des tissus sains. Ce risque a pour origine la production par les cellules de dimères constitués d'un mélange de chaînes $d u T c R$ endogène et de chaînes transgéniques (Figure 2). Les mésappariements conduisent ainsi à une off toxicity qui peut être dramatique lorsque les haTcRT colonisent le tissu cardiaque ou nerveux, leur puissance lytique considérable pouvant entraîner une nécrose des tissus en quelques jours. Les perspectives d'amélioration des haTcRT portent sur l'extinction de l'expression des chaînes de TcR endogènes, des méthodes également applicables aux CAR. Elles portent aussi sur l'exploitation de ce que les chaînes de TcR d'un lymphocyte $T \gamma \delta$ et celles d'un lymphocyte $T \alpha \beta$ ne peuvent s'associer et générer de dimères [40]. Deux options sont ainsi envisagées : transférer un TcR $\alpha \beta$ dans des lymphocytes $T \gamma \delta$ exprimant les chaînes $V \gamma 9 V \delta 2$, ou transférer un TcR $\vee \gamma 9 V \delta 2$ dans des lymphocytes $T \alpha \beta$. Ces deux optimisations ajoutent une seconde spécificité antitumorale à la cellule modifiée : ce TcR $\gamma \delta$ reconnaîtra en effet les phosphoantigènes ${ }^{6}$ qui sont exprimés dans de nombreux types de tumeurs $[2,9,41]^{7}$.

\section{Conclusion}

Après des années de preuves de concept soutenant l'intérêt de l'immunothérapie des cancers, la biologie synthétique ouvre une époque dorée (golden age) dans laquelle la thérapie adoptive par lymphocytes $T$ a une place de choix. Les lymphocytes T sont des «bombes» capables de lyser rapidement des masses tumorales volumineuses. L'avantage des CAR réside dans leur capacité de reconnaissance spécifique d'une cellule tumorale en dehors de tout contexte impliquant le CMH. La restriction due à la présentation par les molécules du CMH est par contre un frein au développement des haTcRT. Mais ces deux types cellulaires sont aussi capables de toxicité aiguë ou chronique (off ou on target) et la sécurité de leur utilisation nécessite de nouveaux développements. Susceptibles de générer

${ }^{6}$ Le TcR des lymphocytes $\gamma \delta$ reconnaît spécifiquement les phosphoantigènes. Ce sont des petits métabolites phosphorylés permettant la biosynthèse des isoprénoïdes par la voie du DOXP (1-déoxy-D-xylulose 5-phosphate) chez les eubactéries ou les protozoaires et par la voie du mévalonate dans les cellules humaines cancéreuses. Le phosphoantigène naturel des lymphocytes $T$ V $\gamma 9 V \delta 2$ dans les cellules tumorales est l'isopentényl pyrophosphate (IPP) dont la biosynthèse est très amplifiée dans les cellules cancéreuses.

${ }^{7}$ C. June annonçait en 2017 ( $9^{\text {th }}$ International Symposium of the clinical use of Cellular Products, Cellular Therapy 2017, 16-17 Mars 2017, Erlangen, Allemagne), que le gain cumulé de survie avait été de 1587 années pour les 468 patients injectés dans la totalité des essais de thérapie par lymphocytes T génétiquement modifiés en Pennsylvanie. 
une auto-immunité, le talon d'Achille de toute immunothérapie, I'utilisation clinique de ces cellules modifiées incite à beaucoup de prudence. Ces deux stratégies bénéficieront certainement d'améliorations portant sur leur spécificité, leur affinité et la maîtrise de leur toxicité, amplifiant ainsi leur efficacité thérapeutique. Ces nouveaux traitements, combinés aux anti-ICP, devraient permettre la révolution tant attendue de l'immunothérapie des cancers: reconnaître spécifiquement la tumeur, amplifier les effecteurs tout en réduisant les problèmes liés à leur potentiel auto-immun. Malgré les difficultés, les CAR entraînent déjà les cliniciens et les investissements des industriels dans leur sillage. $\diamond$

\section{SUMMARY}

Next generation engineered T cells for cell therapy: from lymphoma to solid tumors

FDA approval and French ATU for chimeric antigen receptor (CAR) T cells represent an advanced step in the challenge of immunotherapy to cure cancer. The field of adoptive cell therapy emerged with the discovery that tumor-infiltrating-lymphocytes (TIL) can be used to treat melanoma patients. CAR T cells are engineered by gene transfer to express both receptors that target tumor-associated molecules and killing $T$ cell functions. We report here how several decades of technology combining the specific recognition of an antibody with $T$ cell function have led to the potent activity of CD19-targeting CAR KYMRIAH $^{T M}$ and YESCARTA ${ }^{T M}$ i.e, high remission rates in patients with chemorefractory lymphoma. However, potentially fatal toxicity including cytokine release syndrome and neurotoxicity need next generation developments. Affinity fine-tuning, combinational CARs and guidelines for toxicity management are enhancing the safety of more powerful CAR T. Such CARs are emerging for solid tumor targeting. Synthetic biology approaches leading to personalized cell therapy marks the beginning of a new area. $\diamond$

\section{REMERCIEMENTS}

L'auteure remercie le Cancéropôle Grand Ouest pour son soutien au projet PlasTICO (Plasticité, Tolérance et Immunité dans le cancer de l'Ovaire).

\section{LIENS D'INTÉRÊT}

L'auteure déclare n'avoir aucun lien d'intérêt concernant les données publiées dans cet article.

\section{RéFÉRENCES}

1. Ribas A, Wolchok JD. Cancer immunotherapy using checkpoint blockade. Science 2018 ; 359 : 1350 5 .

2. Catros V, Dessarthe B, Thedrez A, Toutirais 0 . Les récepteurs de nectines/nectines-like DNAM- 1 et CRTAM. Immuno-surveillance ou échappement tumoral ? Med Sci (Paris) 2014 ; 30 : 537-43.

3. Granier C, Gey A, Dariane C, et al. Tim-3. Biomarqueur et cible thérapeutique en cancérologie. Med Sci (Paris) $2018 ; 34: 231-7$.

4. Anderson AC, Joller N, Kuchroo VK. Lag-3, Tim-3, and TIGIT: Co-inhibitory receptors with specialized functions in immune regulation. Immunity $2016 ; 44$ : 989-1004.

5. Pardoll DM. The blockade of immune checkpoints in cancer immunotherapy. Nat Rev Cancer 2012 ; $12: 252-64$.

6. Chen DS, Mellman I. Elements of cancer immunity and the cancer-immune set point. Nature 2017 ; $541: 321-30$.
7. Catros-Quemener V, Bouet $F$, Genetet N. Immunité anti-tumorale et thérapies cellulaires du cancer. Med Sci (Paris) 2003 ; $19: 43-53$.

8. Rosenberg SA, Yang JC, Sherry RM, et al. Durable complete responses in heavily pretreated patients with metastatic melanoma using T-cell transfer immunotherapy. Clin Cancer Res $2011 ; 17:$ 4550-7.

9. Catros V, Toutirais 0 , Bouet $F$, et al. Lymphocytes $T \gamma \delta$ en cancérologie : des lymphocytes tueurs non conventionnels. Med Sci (Paris) $2010 ; 26: 185-91$.

10. Ruella M, Kalos M. Adoptive immunotherapy for cancer. Immunol Rev 2014 ; $257: 14-38$.

11. Castellarin $\mathrm{M}$, Watanabe $\mathrm{K}$, June $\mathrm{CH}$, et al. Driving cars to the clinic for solid tumors. Gene Ther $2018 ; 25: 165-75$.

12. Sadelain M, Riviere I, Riddell S. Therapeutic T cell engineering. Nature 2017 ; $545: 423-31$.

13. Kohl U, Arsenieva S, Holzinger A, Abken H. CAR T cells in trials : Recent achievements and challenges that remain in the production of modified $T$ cells for clinical applications. Hum Gene Ther 2018 ; 29 : 559-68.

14. Gross G, Waks T, Eshhar Z. Expression of immunoglobulin-T-cell receptor chimeric molecules as functional receptors with antibody-type specificity. Proc Natl Acad Sci USA $1989 ; 86$ : 10024-8.

15. Hwu P, Shafer GE, Treisman J, et al. Lysis of ovarian cancer cells by human lymphocytes redirected with a chimeric gene composed of an antibody variable region and the Fc receptor gamma chain. J Exp Med $1993 ; 178$ : 361-6.

16. Cooper LJ, Topp MS, Serrano LM, et al. T-cell clones can be rendered specific for CD19 : toward the selective augmentation of the graft-versus-B-lineage leukemia effect. Blood $2003 ; 101: 1637-44$

17. Pule MA, Savoldo B, Myers GD, et al. Virus-specific T cells engineered to coexpress tumor-specific receptors : persistence and antitumor activity in individuals with neuroblastoma. Nat Med $2008 ; 14$ : 1264-70.

18. Kochenderfer JN, Wilson WH, Janik JE, et al. Eradication of B-lineage cells and regression of lymphoma in a patient treated with autologous T cells genetically engineered to recognize CD19. Blood 2010 ; 116 : 4099-102.

19. Porter DL, Levine BL, Kalos M, et al. Chimeric antigen receptor-modified $T$ cells in chronic lymphoid leukemia. N Engl J Med 2011 ; 365 : 725-33.

20. Brentjens RJ, Davila ML, Riviere I, et al. CD19-targeted T cells rapidly induce molecular remissions in adults with chemotherapy-refractory acute lymphoblastic leukemia. Sci Transl Med 2013; 5 : 177ra38.

21. Davila ML, Riviere I, Wang X, et al. Efficacy and toxicity management of 19-28z CAR T cell therapy in B cell acute lymphoblastic leukemia. Sci Transl Med $2014 ; 6: 224$ ra25.

22. Porter DL, Hwang WT, Frey NV, et al. Chimeric antigen receptor T cells persist and induce sustained remissions in relapsed refractory chronic lymphocytic leukemia. Sci Transl Med $2015 ; 7$ : 303 ral39.

23. Jordan B. Immunothérapie «CAR-T » : une autorisation qui fait date. Med Sci (Paris) 2017 ; 33 : 1003-6.

24. Perales MA, Kebriaei P, Kean LS, Sadelain M.. Biol Blood Marrow Transplant $2018 ; 24: 27-31$.

25. Johnson LA, June CH. Driving gene-engineered T cell immunotherapy of cancer. Cell Res $2017 ; 27: 38-58$.

26. Liu Y, Zhao C, Gao L, et al. Considerations for clinical review of cellular therapy products : perspectives of the china food and drug administration center for drug evaluation. Hum Gene Ther $2018 ; 29$ : 121-7.

27. Lim WA, June $\mathrm{CH}$. The principles of engineering immune cells to treat cancer. Cell 2017 ; $168: 724-40$.

28. O'Rourke DM, Nasrallah MP, Desai A, et al. A single dose of peripherally infused EGFRvIII-directed CAR T cells mediates antigen loss and induces adaptive resistance in patients with recurrent glioblastoma. Sci Transl Med $2017 ; 9$.

29. Posey AD Jr, Schwab RD, Boesteanu AC, et al. Engineered CAR T cells targeting the cancer-associated tn-glycoform of the membrane mucin MUCl control adenocarcinoma. Immunity $2016 ; 44: 1444-54$.

30. Newick K, O'Brien S, Moon $\varepsilon$, Albelda SM. CAR T cell therapy for solid tumors. Annu Rev Med 2017 ; 68 : 139-52.

31. Hudecek M, Sommermeyer D, Kosasih PL, et al. The nonsignaling extracellular spacer domain of chimeric antigen receptors is decisive for in vivo antitumor activity. Cancer Immunol Res $2015 ; 3: 125-35$.

32. Kawalekar OU, RS OC, Fraietta JA, et al. Distinct signaling of coreceptors regulates specific metabolism pathways and impacts memory development in CAR T Cells. Immunity 2016; $44: 712$. 


\section{RÉFÉRENCES}

33. Guedan S, Posey AD, Jr., Shaw $C$, et al. Enhancing CAR T cell persistence through ICOS and 4-IBB costimulation. JCl Insight $2018 ; 3$.

34. Chmielewski M, Abken H. CAR T cells releasing IL-18 convert to T-Bet(high) Fox01(low) effectors that exhibit augmented activity against advanced solid tumors. Cell Rep $2017 ; 21: 3205-19$.

35. Hartmann J, Schussler-Lenz M, Bondanza A, Buchholz CJ. Clinical development of CAR T cellschallenges and opportunities in translating innovative treatment concepts. EMBO Mol Med 2017 $9: 1183-97$

36. Migliorini D, Dietrich Py, Stupp R, et al. CAR T-cell therapies in glioblastoma : a first look. Clin Cancer Res $2018 ; 24: 535-40$.

37. Fraietta JA, Nobles CL, Sammons MA, et al. Disruption of TET2 promotes the therapeutic efficacy of CD19-targeted T cells. Nature 2018 ; $558: 307-12$.
38. Ruella M, Kenderian SS. Next-generation chimeric antigen receptor T-cell therapy : going off the shelf. BioDrugs $2017 ; 31: 473-81$.

39. Thedrez A, Lavoue V, Dessarthe B, et al. A quantitative deficiency in peripheral blood Vgamma9Vdelta2 cells is a negative prognostic biomarker in ovarian cancer patients. PLoS One 2013 ; 8 : e63322.

40. Marcu-Malina V, Heijhuurs S, van Buuren M, et al. Redirecting alphabeta T cells against cancer cells by transfer of a broadly tumor-reactive gammadeltaT-cell receptor. Blood $2011 ; 118: 50-9$.

41. Dessarthe $B$, Thedrez A, Latouche JB, et al. CRTAM receptor engagement by Necl-2 on tumor cells triggers cell death of activated vgamma9vdelta2 T Cells. J immunol $2013 ; 190: 4868-76$.

\section{TIRÉS À PART}

\section{Catros}

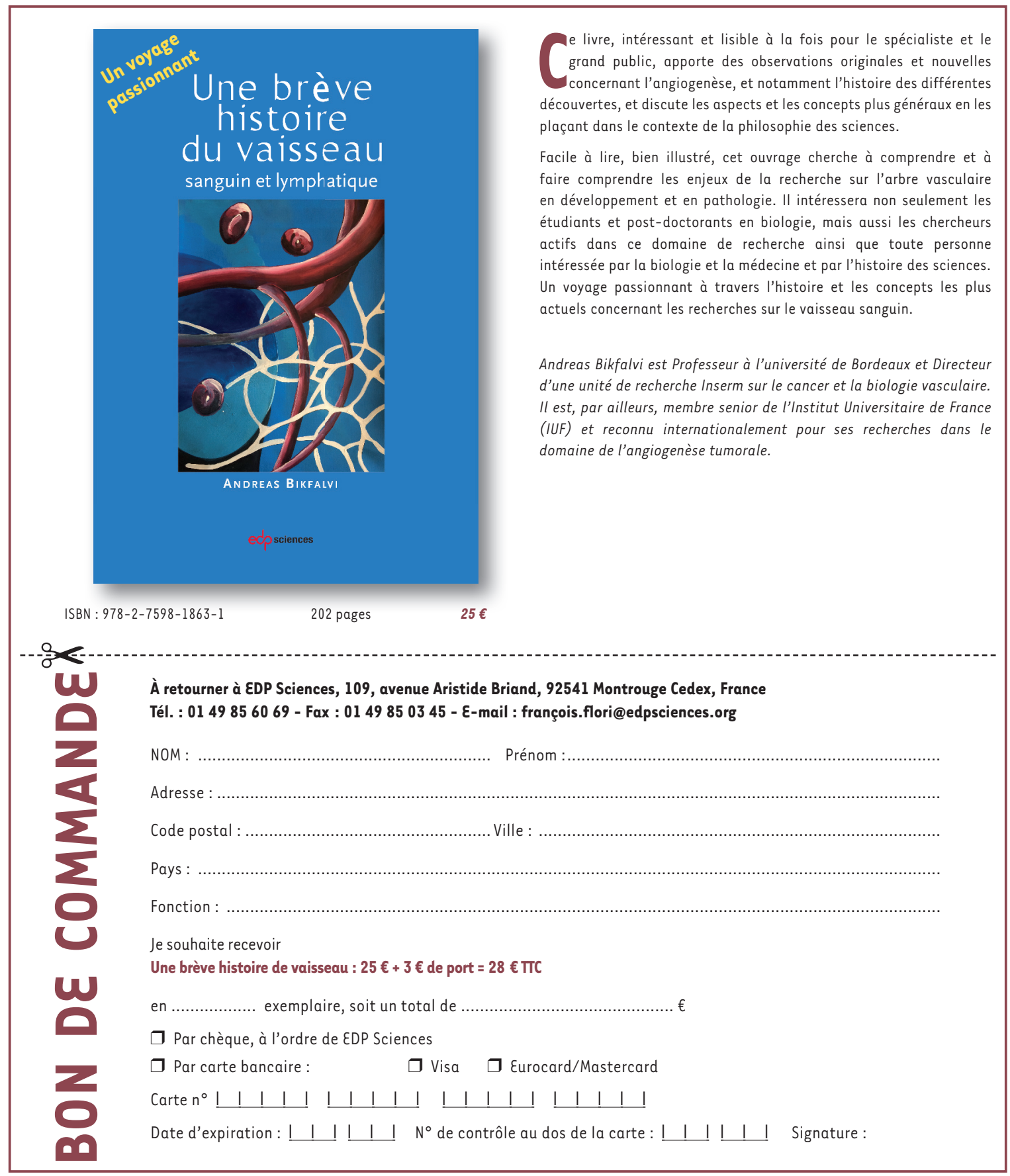

